\title{
TRANSIENT MOTION OF A LINE LOAD ON THE SURFACE OF AN ELASTIC HALF-SPACE*
}

\author{
BY \\ DANG DINH ANG \\ Guggenheim Aeronautical Laboratory, California Institute of Technology
}

Abstract. The present paper studies the wave patterns generated in an elastic half-space by a line load moving on its surface with a velocity varying as a step function of time. The solution given in closed form is obtained by means of Fourier integral equations techniques following a Laplace transformation with respect to the time variable. The inversion of the Laplace transforms is based on a trick due to Cagniard and De Hoop.

Introduction. For the past few years, people have been interested in the effects that moving blast waves on the surface of the earth exert on its interior and in particular on underground structures. The problem has been considered by Sneddon [1] and independently by Cole and Huth [2], who treated the steady motion of a line load on the surface of an elastic half-space. Although it is clear that, far enough from the starting position of the load, the motion can be considered as essentially steady, there is one important feature that is not exhibited by a steady state solution, i.e., the "resonance" effect present in a motion at a velocity approaching the Rayleigh wave velocity. The present paper will take account of the latter effect by considering the transient motion of a line load at a velocity varying as a step function of the time.

The initial and boundary value problem. Let a Cartesian system of coordinate. $x, y, z$ be defined such that the elastic half-space is represented by $y \geq 0$, that the axis of the line load is in the $z$-direction and that its initial position coincides with the $z$-axis. The strength of the load is assumed constant so that the problem is a two-dimensional one in $x, y$. Furthermore, since the medium extends indefinitely in the $z$-direction, the problem is one of plane strain. Then, if $u$ and $v$ denote the components of the displacement in the $x$ - and $y$-directions respectively, it can be shown [3] that the equations of elastic motion are satisfied if

$$
u=-\frac{\partial \Phi}{\partial x}+\frac{\partial \Psi}{\partial y}, \quad v=-\frac{\partial \Phi}{\partial y}-\frac{\partial \Psi}{\partial x},
$$

where $\Phi$ and $\Psi$ are solutions of

$$
\nabla^{2} \Phi=v_{1}^{2} \frac{\partial^{2} \Phi}{\partial t^{2}}, \quad \nabla^{2} \Psi=v_{2}^{2} \frac{\partial^{2} \Psi}{\partial t^{2}} .
$$

In (2), $v_{1}^{2}$ and $v_{2}^{2}$ are defined as

$$
v_{1}^{2}=\rho /(\lambda+2 \mu), \quad v_{2}^{2}=\rho / \mu,
$$

where $\rho$ is the density of the material, and $\lambda, \mu$ its Lamé constants.

\footnotetext{
${ }^{*}$ Received March 10, 1959; revised manuscript received August 28, 1959. This investigation was supported in part by the United States Navy Bureau of Ordnance through the U. S. Naval Ordnance Test Station, China Lake, Calif.
} 
The initial and boundary conditions are:

$$
\begin{array}{rlrl}
\left\{\begin{array}{rlrl}
\tau_{y y} & =-\delta\left(x-c_{0}^{-1} t\right) y & =0, x>0 \\
& =0 & y & =0, x<0
\end{array}\right. & \\
\tau_{x y} & =0 & y & =0, \text { for all } x,
\end{array}
$$

where $\tau_{y y}$ and $\tau_{x y}$ are respectively the normal and shear stresses at the surfaces of constant $y, \delta$ is a Dirac delta-function, and $c_{0}^{-1}$ is the velocity of the moving load assumed to be a constant. We thus consider a normal load only, but it will be seen that the general case could be handled just as easily, and that the characteristic features of the wave patterns of direct interest here are not changed if we add to the load a tangential component. To the conditions (4)-(5), we must, of course, add the condition that the waves be outgoing waves. To complete the formulation of the problem, we require the wave functions $\Phi$ and $\Psi$ to be continuously differentiable at least twice everywhere except at the the wave fronts, and the displacement to be a continuous function of the space coordinates everywhere inside the solid.

The method of solution consists in applying a Laplace transformation to the wave equations (2), and then expressing the solutions of the resulting equations as superpositions of "plane waves" with the amplitude spectra as the unknowns. Thus, under a Laplace transformation defined in the usual manner, the Eqs. (2) become:

$$
\nabla^{2} \Phi^{*}=v_{1}^{2} p^{2} \Phi^{*}, \quad \nabla^{2} \Psi^{*}=v_{2}^{2} p^{2} \Psi^{*},
$$

where $p$ is the parameter of the transformation and the superscript* indicates a Laplace transform. Let the solutions of (6) be of the forms:

$$
\begin{aligned}
& \Phi^{*}=\int_{-\infty}^{\infty} P(s) \exp \left\{-p\left(s^{2}+v_{1}^{2}\right)^{1 / 2} y+i p s x\right\} d s, \\
& \Psi^{*}=\int_{-\infty}^{\infty} Q(s) \exp \left\{-p\left(s^{2}+v_{2}^{2}\right)^{1 / 2} y+i p s x\right\} d s,
\end{aligned}
$$

where $P(s)$ and $Q(s)$ are unknown functions and the paths of integration are along the real axis. The functions $\left(s^{2}+v_{1,2}^{2}\right)^{1 / 2}$ are given non-negative real parts along the path of integration; this can be achieved by defining their branch cuts as follows: $\operatorname{Re} s=0$, $v_{1,2}<|\operatorname{Im} s|<\infty$ respectively. Finally, the parameter $p$ in (7)-(8) is taken to be a real and positive quantity of magnitude sufficiently large to insure the convergence of the integrals.

The problem of course is to determine the functions $P(s)$ and $Q(s)$ from the boundary conditions. Now, the conditions of (4) and (5) can be expressed in terms of $\Phi$ and $\Psi$ using (1) and the well-known strain-displacement and stress-strain relations. Assuming that (7) and (8) can be differentiated under the integral signs, we accordingly obtain for the Laplace transforms of (4)-(5)

$$
\begin{aligned}
& \int_{-\infty}^{\infty}\left\{\left(s^{2}+v_{2}^{2} / 2\right) P(s)-i s\left(s^{2}+v_{1}^{2}\right)^{1 / 2} Q(s)\right\} \exp (i p s x) d s
\end{aligned}
$$

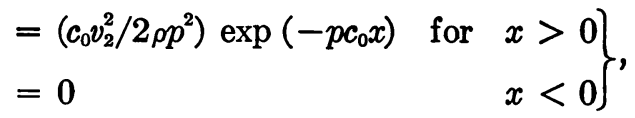




$$
\int_{-\infty}^{\infty}\left\{2 i s\left(s^{2}+v_{1}^{2}\right)^{1 / 2} P(s)+\left(2 s^{2}+v_{2}^{2}\right) Q(s)\right\} \exp (i p s x) d s=0
$$

the latter equation holding for all $x$. Equation (10) is evidently satisfied if the integrand is identically zero, i.e., if

$$
P(s)=\left(s^{2}+v_{2}^{2} / 2\right) R(s), \quad Q(s)=-i s\left(s^{2}+v_{1}^{2}\right)^{1 / 2} R(s),
$$

where $R(s)$ is still largely arbitrary. Substituting for $P(s)$ and $Q(s)$ into (9) gives:

$$
\left.\begin{array}{rlrl}
\int_{-\infty}^{\infty} F(s) R(s) \exp (i p s x) d s & =\left(A_{0} / p^{2}\right) \exp \left(-p c_{0} x\right), & & x>0 \\
& =0 & & x<0
\end{array}\right\},
$$

where

$$
A_{0} \equiv c_{0} v_{2}^{2} / 2 \rho, \quad F(s) \equiv\left(s^{2}+v_{2}^{2} / 2\right)^{2}-s^{2}\left(s^{2}+v_{1}^{2}\right)^{1 / 2}\left(s^{2}+v_{2}^{2}\right)^{1 / 2} .
$$

The solution of (12) is immediate

$$
R(s)=\left(A_{0} / 2 \pi i p^{2}\right)\left\{\left(s-i c_{0}\right) F(s)\right\}^{-1} .
$$

From (11) and (14), the expressions for $P(s)$ and $Q(s)$ can be deduced and the problem is formally solved. It is not difficult to show that this solution converges and is the actual solution of the boundary value problem.

The stress wave patterns. It remains to find the inverse transform of the solutions. The Laplace transforms of the stresses are given in terms of integrals of the form

$$
I^{*}=\int_{-\infty}^{\infty} K(s) \exp \left\{-p\left(s^{2}+v^{2}\right)^{1 / 2} y+i p s x\right\} d s,
$$

where $v=v_{1,2}$ and $K(s)$ is some algebraic function of $s$ only. The present inversion of (15) back to the $t$-plane is based on a trick originally due to Cagniard [4] and modified by De Hoop [5]. The trick consists in so deforming the path of integration that $I^{*}$ is reduced to the form

$$
I^{*}=\int_{0}^{\infty} J(t ; x, y) e^{-p t} d t,
$$

where $t$ is real and $J(t ; x, y)$ is some suitable function. The inverse of $I^{*}$ can thus be obtained by inspection. In the course of the deformation of the path of integration, it is essential to know all the singularities of $K(s)$. These can be recognized by inspection, with the exception of the poles $s= \pm$ is $_{R}, s_{R}>v_{2}, \pm$ is ${ }_{R}$ being the zeros* of $F(s)$ defined in (13). Once the singularities of $K(s)$ are known, it only remains to follow steps quite similar to those taken in [6] where the inverse transforms of integrals of the form (15) have been found. We refer the reader to [6] for these details and are content to state here the final expressions for the stresses corresponding to a velocity of the load smaller** than both velocities of sound in the solid

\footnotetext{
*Thus $s_{R}{ }^{-1}$ is the well-known Rayleigh wave velocity.

**The case of a load velocity greater than either or both velocities of sound could be handled just as easily but is omitted for simplicity.
} 


$$
\begin{aligned}
& \tau_{y \nu}=-\frac{c_{0}}{\pi r} H\left(t-v_{1} r\right)\left\{\frac{t \sin \theta}{\left(t^{2}-v_{1}^{2} r^{2}\right)^{1 / 2}} \operatorname{Re} \frac{M_{1}\left(s_{1}\right)}{\left(c_{0}+i s_{1}\right)\left(s_{R}+i s_{1}\right)}\right. \\
& \left.-\operatorname{Im} \frac{M_{1}\left(s_{1}\right) \cos \theta}{\left(c_{0}+i s_{1}\right)\left(s_{R}+i s_{1}\right)}\right\}+\Delta \tau, \\
& \Delta \tau \equiv \frac{c_{0}}{\pi r} H\left(t-v_{2} r\right)\left\{\frac{t \sin \theta}{\left(t^{2}-v_{2}^{2} r^{2}\right)^{1 / 2}} \operatorname{Re} \frac{M_{2}\left(s_{2}\right)}{\left(c_{0}+i s_{2}\right)\left(s_{R}+i s_{2}\right)}-\operatorname{Im} \frac{M_{2}\left(s_{2}\right) \cos \theta}{\left(c_{0}+i s_{2}\right)\left(s_{R}+i s_{2}\right)}\right\} \\
& -\frac{c_{0}}{\pi r} f(t) g(\theta)\left\{ \pm \frac{t \sin \theta}{\left(v_{2}^{2} r^{2}-t^{2}\right)^{1 / 2}}+\cos \theta\right\} \cdot \operatorname{Im} \frac{M_{2}\left(\omega_{\star}\right)}{\left(c_{0}+i \omega_{\star}\right)\left(s_{R}+i \omega_{\star}\right)}, \\
& \tau_{x x}=\frac{c_{0}}{\pi r} H\left(t-v_{1} r\right)\left\{\frac{t \sin \theta}{\left(t^{2}-v_{1}^{2} r^{2}\right)^{1 / 2}} \operatorname{Re} \frac{N_{1}\left(s_{1}\right)}{\left(c_{0}+i s_{1}\right)\left(s_{R}+i s_{1}\right)}\right. \\
& \left.-\operatorname{Im} \frac{N_{1}\left(s_{1}\right) \cos \theta}{\left(c_{0}+i s_{1}\right)\left(s_{R}+i s_{1}\right)}\right\}-\Delta \tau, \\
& \tau_{x y}=-\frac{c_{0}}{\pi r} H\left(t-v_{1} r\right)\left\{\frac{t \sin \theta}{\left(t^{2}-v_{1}^{2} r^{2}\right)^{1 / 2}} \operatorname{Re} \frac{N_{2}\left(s_{1}\right)}{\left(c_{0}+i s_{1}\right)\left(s_{R}+i s_{1}\right)}\right. \\
& \left.-\operatorname{Im} \frac{N_{2}\left(s_{1}\right) \cos \theta}{\left(c_{0}+i s_{1}\right)\left(s_{R}+i s_{1}\right)}\right\} \\
& +\frac{c_{0}}{\pi r} H\left(t-v_{2} r\right)\left\{\frac{t \sin \theta}{\left(t^{2}-v_{2}^{2} r^{2}\right)^{1 / 2}} \operatorname{Re} \frac{N_{2}\left(s_{2}\right)}{\left(c_{0}+i s_{2}\right)\left(s_{R}+i s_{2}\right)}\right. \\
& \left.-\operatorname{Im} \frac{N_{2}\left(s_{2}\right) \cos \theta}{\left(c_{0}+i s_{2}\right)\left(s_{R}+i s_{2}\right)}\right\} \\
& -\frac{c_{0}}{\pi r} f(t) g(\theta)\left\{ \pm \frac{t \sin \theta}{\left(v_{2}^{2} r^{2}-t^{2}\right)^{1 / 2}}+\cos \theta\right\} \operatorname{Im} \frac{N_{2}\left(\omega_{ \pm}\right)}{\left(c_{0}+i \omega_{\star}\right)\left(s_{R}+i \omega_{\star}\right)},
\end{aligned}
$$

where

$$
\begin{gathered}
r=\left(x^{2}+y^{2}\right)^{1 / 2}, \quad \theta=\tan ^{-1}(y / x), \quad 0 \leq \theta \leq \pi, \\
s_{1,2}=\left(t^{2} / r^{2}-v_{1,2}^{2}\right)^{1 / 2} \sin \theta+i(t / r) \cos \theta, \\
M_{1}(s)=\left(s^{2}+v_{2}^{2} / 2\right)^{2}\left(s_{R}+i s\right) / F(s),
\end{gathered}
$$

$F(s)$ being defined in (13)

$$
\begin{gathered}
M_{2}(s)=s^{2}\left(s^{2}+v_{1}^{2}\right)^{1 / 2}\left(s^{2}+v_{2}^{2}\right)^{1 / 2}\left(s_{R}+i s\right) / F(s), \\
\left(s_{1,2}^{2}+v_{1,2}^{2}\right)^{1 / 2}=\left\{t \sin \theta+i\left(t^{2}-v_{1,2}^{2} r^{2}\right)^{1 / 2} \cos \theta\right\} / r \\
\left(s_{1,2}^{2}+v_{2,1}^{2}\right)^{1 / 2}=\left\{\frac{\left(A_{1,2}^{2}+B_{1,2}^{2}\right)^{1 / 2}+A_{1,2}}{2}\right\}^{1 / 2} \pm i\left\{\frac{\left(A_{1,2}^{2}+B_{1,2}^{2}\right)^{1 / 2}-A_{1,2}}{2}\right\}^{1 / 2}, \\
A_{1,2} \equiv\left(t^{2} / r^{2}\right)\left(\sin ^{2} \theta-\cos ^{2} \theta\right)+v_{2,1}^{2}-v_{1,2}^{2} \sin ^{2} \theta \\
B_{1,2} \equiv\left(t^{2} / r^{2}-v_{1,2}^{2}\right)^{1 / 2}(t / r) \sin 2 \theta,
\end{gathered}
$$

the \pm signs in (25) corresponding to $\cos \theta>0$ and $\cos \theta<0$ respectively. 


$$
\begin{aligned}
& {\left[\begin{array}{rl}
f(t)= & 1 t_{s} \leq t \leq v_{2} r \\
& t_{s} \equiv v_{2} r|\cos \theta|+\left(v_{2}^{2}-v_{1}^{2}\right)^{1 / 2} r \sin \theta \\
= & 0 \text { otherwise }
\end{array}\right.} \\
& {\left[\begin{array}{c}
g(\theta)=10 \leq \theta \leq 0_{S} \text { or } \pi-\theta_{S} \leq 0 \leq \pi \\
\theta_{S} \equiv \cos ^{-1}\left(v_{1} / v_{2}\right) \\
=0 \text { otherwise }
\end{array}\right.} \\
& \omega_{\star}=i\left\{\mp\left(v_{2}^{2}-t^{2} / r^{2}\right)^{1 / 2} \sin \theta+(t / r) \cos \theta\right\},
\end{aligned}
$$

$\omega_{-}$corresponding to $0 \leq \theta \leq \theta_{S}$ and $\pi-0_{S} \leq \theta \leq \pi$ respectively.

$$
\begin{aligned}
& \left(\omega_{-}^{2}+v_{1}^{2}\right)^{1 / 2}= \pm i\left(\left|\omega_{-}\right|^{2}-v_{1}^{2}\right)^{1 / 2} \\
& \left(\omega_{-}^{2}+v_{2}^{2}\right)^{1 / 2}=\left(v_{2}^{2}-\left|\omega_{+}\right|^{2}\right)^{1 / 2} \\
N_{1}(s)= & \left(s^{2}+v_{1}^{2}-v_{2}^{2} / 2\right)\left(s^{2}+v_{2}^{2} / 2\right)\left(s_{R}+i s\right) / F(s), \\
N_{2}(s)= & i s\left(s^{2}+v_{1}^{2}\right)^{1 / 2}\left(s^{2}+v_{2}^{2} / 2\right)\left(s_{R}+i s\right) / F(s) .
\end{aligned}
$$

In the above relations the square roots of real quantities are taken as positive.

From (17)-(19), it is clear that the wave patterns consist of two cylindrical waves radiating from the origin $r=0$ with velocities $v_{1}^{-1}$ and $v_{2}^{-1}$ respectively, two head waves whose fronts are tangential to the shear cylindrical wave-the head waves are represented e.g. in $\Delta \tau$ in (18) - the two surface waves propagating in the positive and negative $x$-directions respectively. The complete picture is shown in the figure below.

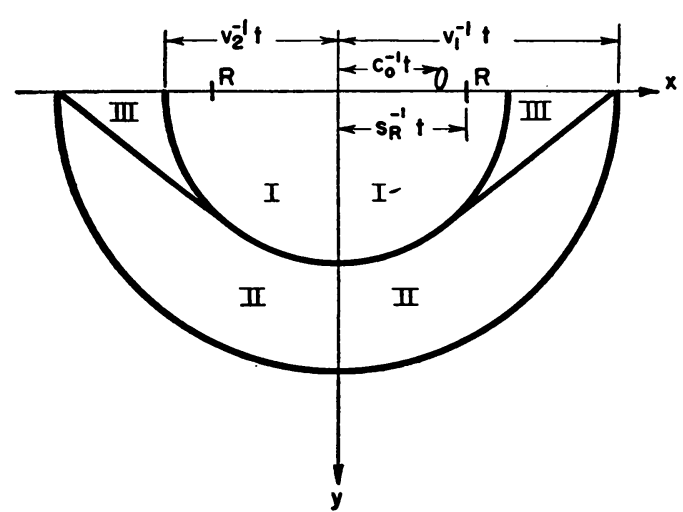

Stress wave patterns. I. Shear wave, II. Dilatation wave, III. Head waves, R. Rayleigh wave.

It can be verified that the stresses are of the order $\left(v r^{2}-t^{2}\right)^{-1 / 2}$, for $v r \rightarrow t, v=v_{1,2}$. This singularity is, of course, integrable and does not affect the continuity of the displacements. The resonance effect anticipated in the Introduction results from the 
presence of the factors $\left(c_{0}+i s\right)^{-1}\left(s_{R}+i s\right)^{-1}, s=s_{1,2}$, in the expressions (17)-(19) since $-i s_{R}$ is not a zero of the functions $M(s), N(s)$ defined above.

\section{REFERENCES}

1. I. N. Sneddon, The stress produced by a pulse of pressure moving along the surface of a semi-infinite solid, R. C. Circ. Mat. Palermo, 2, 57-62 (1952)

2. J. D. Cole and J. Huth, Stresses produced in a half-plane by moving loads, J. Appl. Mech. 25, 433-36 (Dec. 1958)

3. P. M. Morse and H. Feshbach, Methods of theoretical physics, vol. 1, McGraw-Hill, 1953, pp. 142-148

4. L. Cagniard, Reflexion et refraction des ondes seismiques progressives, Gauthier-Villars, Paris, 1939, p. 55

5. A. T. De Hopp, Representation theorems for the displacement in an elastic solid and their application to elastodynamic diffraction, Thesis, Technische Hogeschool Te Delft, pp. 35-55, 1958

6. D. D. Ang, Elastic waves generated by a force moving along a crack, J. Math. Phys. 38, 246-256 (1960) 